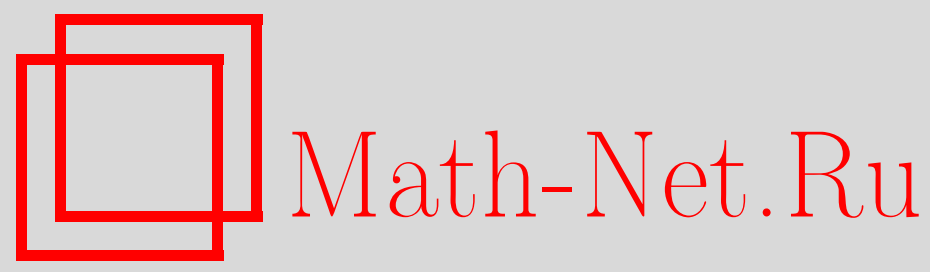

О. Н. Григорьев, М. В. Карасёв, Динамическое уравнение для квантового произведения в афинных координатах на симплектическом пространстве, Матем. заметки, 2005, том 77 , выпуск $1,42-52$

DOI: https://doi.org/10.4213/mzm2467

Использование Общероссийского математического портала Math-Net.Ru подразумевает, что вы прочитали и согласны с пользовательским соглашением http://www.mathnet.ru/rus/agreement

Параметры загрузки:

IP: 3.80 .181 .102

26 апреля 2023 г., 14:50:59

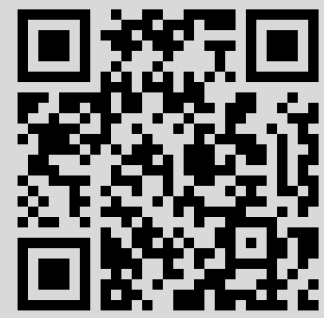




\title{
ДИНАМИЧЕСКОЕ УРАВНЕНИЕ ДЛЯ КВАНТОВОГО ПРОИЗВЕДЕНИЯ В АФИННЫХ КООРДИНАТАХ \\ НА СИМПЛЕКТИЧЕСКОМ ПРОСТРАНСТВЕ
}

\section{О.Н. Григорьев, М.В. Карасев}

\begin{abstract}
Выводится динамическая система, описывающая ассоциативное некоммутативное произведение функций на симплектическом пространстве. Она явно решается в квазиклассическом приближении.

Библиографоия: 16 названий.
\end{abstract}

1. Введение. Как известно, описание квантовых систем основано на алгебрах операторов, действующих в гильбертовом пространстве. Очень продуктивной является идея представить алгебру операторов с помощью функций, определенных на некотором многообразии, т.е. построить отображение $\mathrm{Op}(f) \rightarrow f$ из пространства операторов в пространство функций (символов). При этом композиции операторов $\mathrm{Op}(f) \mathrm{Op}(g)$ будет соответствовать символ $f * g$, который называется квантовым произведением символов $f$ и $g$. Это произведение некоммутативно, так как соответствующие операторыне перестановочны друг с другом. Кроме того, поскольку композиция операторов ассоциативна, условие ассоциативности переносится и на квантовое произведение (см. [1]-[4]).

Мы рассмотрим в данной заметке процедуру построения квантового произведения, используя уравнения внутренней динамики фазового многообразия. В качестве многообразия возьмем пространство $\mathfrak{X} \approx \mathbb{R}^{2 n}$, наделенное симплектической формой $\omega$ и симплектической связностью (без кручения) $\Gamma$.

Следуя подходу деформационного квантования [1], мы рассматриваем квантовое произведение $*$ над $\mathfrak{X}$, которое зависит от параметра деформации $\hbar>0$ так, что

$$
f * g=f g-\frac{i \hbar}{2} f \underset{\leftarrow}{\nabla} \underset{\rightarrow}{\nabla} g-\frac{\hbar^{2}}{8} f(\underset{\leftarrow}{\nabla} \underset{\rightarrow}{\rightarrow})^{2} g+O\left(\hbar^{3}\right) ;
$$

здесь $\Psi=\omega^{-1}$ - пуассонов тензор, соответствующий форме $\omega$, ковариантные производные $\nabla$ задаются связностью $\Gamma$, а нижние стрелки указывают влево или вправо действует ковариантная производная.

Асимптотика (1.1) (и ее продолжение до любой точности $O\left(\hbar^{N}\right)$ ) слабая, т.е. она имеется только на гладких $\hbar$-независимых функциях $f, g$. В то же время, интерес представляют более общие формулы для *, например, в виде интеграла

$$
(f * g)(u)=\frac{1}{(2 \pi \hbar)^{2 n}} \int K_{x, y}^{\hbar}(u) f(x) g(y) d x d y
$$


относительно стандартной меры на $\mathfrak{X}$. Здесь интегральное ядро $K^{\hbar}$ сингулярно при $\hbar \rightarrow 0$ так, что в слабом смысле (1.2) переходит в (1.1); в частности, имеется слабый предел

$$
\lim _{\hbar \rightarrow 0} \frac{1}{(2 \pi \hbar)^{2 n}} K_{x, y}^{\hbar}=\delta_{x} \delta_{y} .
$$

Но, главное, формула типа (1.2) может быть применена и к $\hbar$-зависимым функциям, например, к быстро осциллирующим вида $f(x) \sim e^{\frac{i}{\hbar} S(x)} \varphi(x)$, что делает возможным использование произведения (1.2) в квантово-динамических задачах с фазовым пространством $\mathfrak{X}$.

Возникает проблема вычисления ядра $K^{\hbar}$. На решение этой проблемы в квазиклассическом приближении направлена теория асимптотического квантования, инициированная в [3].

Известно, что для произвольного пуассонова многообразия $\mathfrak{X}$ функции $K^{\hbar}$ соответствует лагранжево подмногообразие в некотором симплектическом пространстве $\mathscr{E} \times \mathscr{E} \times \mathscr{E}$ над конфигурационньм пространством $\mathfrak{X} \times \mathfrak{X} \times \mathfrak{X}$. Это лагранжево подмногообразие - график операции умножения симплектического группоида $\mathscr{E}$ над $\mathfrak{X}$ [4]-[6]. Поэтому квазиклассическую асимптотику ядра $K^{\hbar}$ в общем пуассоновом случае можно вычислять с помощью модификации теории канонического оператора Маслова на случай общих симплектических многообразий [3], [4].

Чтобы реализовать этот подход, необходимо иметь (псевдо-) дифференциальные уравнения для $K^{\hbar}$. Такие уравнения были найдены в [7], что дало возможность асимптотически вычислить $K^{\hbar}[4]$. Однако в высших приближениях получаюшиеся формулы чрезвычайно громоздки и не "геометричны".

В случае, когда $\mathfrak{X}$ - симплектическое многообразие, существенное упрощение дает идея, предложенная в [8]: записать для ядра $K^{\hbar}$ динамическое уравнение типа Шрёдингера

$$
i \hbar \frac{\partial}{\partial y} K_{x, y}^{\hbar}=\mathscr{H}_{y}^{\hbar} * K_{x, y}^{\hbar}
$$

с некоторым гамильтонианом $\mathscr{H}_{y}^{\hbar}$, зависяшим от “времени" $y \in \mathfrak{X}$. Получающаяся динамическая система нелинейна, поскольку произведение * в правой части (1.3) задается ядром $K^{\hbar}$ по формуле (1.2).

Гамильтониан $\mathscr{H}_{y}^{\hbar}$ является 1-формой на $\mathfrak{X}$, коэффициентами которой служат функции на $\mathfrak{X}$ :

$$
\mathscr{H}_{y}^{\hbar}(x)=\mathscr{H}_{y}^{\hbar}(x)_{j} d y^{j}, \quad x \in \mathfrak{X}
$$

Эти коэффициенты регулярно зависят от $\hbar$ :

$$
\mathscr{H}^{\hbar}=\mathscr{H}^{(0)}-i \hbar \mathscr{H}^{(1)}+\hbar^{2} \mathscr{H}^{(2)}+\cdots
$$

где $\mathscr{H}^{(1)}, \mathscr{H}^{(2)}, \ldots$ - вешественные квантовые поправки к старшей части $\mathscr{H}^{(0)}$. Старшая часть $\mathscr{H}^{(0)}=\mathscr{H}_{y}^{(0)}(x)$ в $(1.4)$, по крайней мере в окрестности диагонали $x=y$, задается парой $\omega, \Gamma ;$ см. подробнее [8].

В [8] было показано, что при дополнительном условии вещественности $\mathscr{H}^{\hbar}$ уравнение (1.3) эффективно и геометрически-инвариантно решается в квазиклассическом приближении. 
В данной заметке, не предполагая вещественности, мы вычислим гамильтониан $\mathscr{H}_{y}^{\hbar}$ (1.4) в специальных $\omega$-аффинных координатах на $\mathfrak{X}$, и найдем главньй член ВКБ-асимптотики ядра $K^{\hbar}$, учитьваюший мнимую квантовую поправку $\mathscr{H}^{(1)}$ в $(1.4)$. В работе [9], а также в пункте 5 ниже разбираются примеры, где такая мнимая поправка действительно появляется.

2. Аффинные координаты и вейлевское произведение. Следуя [8], мы будем назьвать систему координат $x_{1}, \ldots, x_{m}$ на $\mathfrak{X} \approx \mathbb{R}^{m}(m=2 n) \omega$-аффинной, или просто аффинной, если для любой тройки индексов $j, k, l$ выполнено равенство

$$
\underset{(j, k, l)}{\mathfrak{S}} \omega_{j s} \Gamma_{k l}^{s}=0
$$

здесь $\omega_{j s}$ - компоненты формы $\omega$, a $\Gamma_{k l}^{s}-$ символы Кристофеля связности $\Gamma$ в данных координатах; по повторяющимся индексам подразумевается суммирование от 1 до $m$, a знак $\mathfrak{S}$ означает сумму по всем циклическим перестановкам данной тройки индексов.

В аффинных координатах связность Г задается формулой

$$
\Gamma_{i j}^{k}=\frac{1}{3} \Psi^{k s}\left(\partial_{i} \omega_{s j}+\partial_{j} \omega_{s i}\right) .
$$

Произведение * называется вейлевским [10] по отношению к данной $\omega$-аффинной системе координат, если оно инвариантно относительно вейлевской симметризации, т.е.

$$
x^{\alpha} \stackrel{\text { def }}{=}\left\langle x^{\alpha}\right\rangle \quad \forall \alpha=\left(\alpha_{1}, \ldots, \alpha_{m}\right) .
$$

Здесь слева стоит обычный моном $x^{\alpha}=x_{1}^{\alpha_{1}} \ldots x_{m}^{\alpha_{m}}$, а справа стоит моном в $*$-алгебре, симметризованньй по Вейлю:

$$
\left.\left\langle x^{\alpha}\right\rangle \stackrel{\text { def }}{=}\left(\frac{\partial}{\partial u}\right)^{\alpha} \underbrace{(u x) * \cdots *(u x)}_{|\alpha|}\right|_{u=0},
$$

где обозначено $u x=u_{1} x_{1}+\cdots+u_{m} x_{m}$. Для вейлевского произведения в [7] была указана процедура построения операторов левого и правого регулярного представления в виде:

$$
x_{j} * \equiv L^{j}=\ell_{\hbar}^{j}\left(\stackrel{2}{x},-i \hbar \frac{\partial}{\partial x}\right), \quad * x_{j} \equiv R^{j}=\bar{L}^{j} .
$$

Асимптотика символов $\ell_{\hbar}$ явно вычисляется во всех порядках по $\hbar$ :

$$
\ell_{\hbar}(x, p)=\ell(x, p)+i \hbar \mu(x, p)+\cdots .
$$

Здесь главная часть символов дается формулами [11], [8]

$$
\ell_{j}(x, p)=\Lambda\left(p \Psi(x) \frac{\partial}{\partial x}\right) x_{j}
$$

где $\Lambda(\xi)=\xi /\left(e^{\xi}-1\right)$. Вектор-функции $\ell(x, p)$ и $r(x, p)=\ell(x,-p)$ задают пуассоново бирасслоение $\ell \times r: \mathscr{E} \rightarrow \mathfrak{X} \times \mathfrak{X}^{(-)}$фазового пространства (симплектического группоида) над $\mathfrak{X}$ в прямое произведение двух копий $\mathfrak{X}$, наделенное разностью скобок Пуассона на сомножителях (см. детали в [4], [5]). Первая квантовая поправка в (2.4) имеет вид

$$
\mu_{j}(x, p)=\frac{1}{2} \frac{\partial \widetilde{P}_{p}^{k}}{\partial p_{s}}(\ell(x, p)) \frac{\partial^{2} \ell_{j}(x, p)}{\partial x_{s} \partial x_{k}},
$$

где $\widetilde{P}_{p}^{k}=P_{-p}^{k}$, а функция $x=P_{p}(\xi)$ является решением неявного уравнения $\xi=r(x, p)$ относительно $x$. 
3. Внутренняя квантовая динамика. Теперь мы выведем динамическое уравнение (1.3) для ядра вейлевского *-произведения, т.е. найдем гамильтониан $\mathscr{H}^{\hbar}$ в аффинных координатах. Будем исходить из свойства ассоциативности

$$
(f * g) * h=f *(g * h) .
$$

Используя регулярное представление, в силу $(2.3)$ для всех $j=1, \ldots, 2 n$ имеем

$$
f * x_{j} * g=f *\left(x_{j} * g\right)=f * L^{j}(g) .
$$

С другой стороны, в силу ассоциативности это же выражение записывается в виде

$$
f * x_{j} * g=\left(f * x_{j}\right) * g=R^{j}(f) * g .
$$

Используя теперь интегральную формулу (1.2), получим следующее интегральное равенство:

$$
\int K_{x, y}^{\hbar}(u) R^{j}(f)(x) g(y) d x d y=\int K_{x, y}^{\hbar}(u) f(x) L^{j}(g)(y) d x d y .
$$

Перебрасьвая операторы регулярного представления на ядро, получим

$$
\int\left(R_{x}^{* j} K_{x, y}^{\hbar}\right)(u) f(x) g(y) d x d y=\int\left(L_{y}^{* j} K_{x, y}^{\hbar}\right)(u) f(x) g(y) d x d y
$$

Нижний индекс у операторов означает переменную, по которой они действуют. Поскольку $f$ и $g$ - произвольные функции, получаем отсюда следующие уравнения на интегральное ядро $K$ :

$$
\left(L_{y}^{* j} K_{x, y}^{\hbar}\right)=\left(R_{x}^{* j} K_{x, y}^{\hbar}\right) .
$$

Действуя на обе части последнего равенства операторами левого регулярного представления, будем иметь (операторы $L$ и $R$ действуют по разным переменным, а потому коммутируют)

$$
L_{y}^{i *}\left(L_{y}^{j *} K_{x, y}^{\hbar}\right)=L_{y}^{i *}\left(R_{x}^{j *} K_{x, y}^{\hbar}\right)=R_{x}^{j *}\left(L_{y}^{i *} K_{x, y}^{\hbar}\right)=R_{x}^{i *}\left(R_{x}^{j *} K_{x, y}^{\hbar}\right) .
$$

Отсюда по индукции для любого полинома $\mathscr{P}$ можно записать следующее соотношение:

$$
\mathscr{P}\left(L_{y}^{*}\right) K_{x, y}^{\hbar}=\mathscr{P}\left(R_{x}^{*}\right) K_{x, y}^{\hbar}
$$

Однако можно считать, что коэффициенты полинома $\mathscr{P}$ - это функции от переменной $y$, если задать порядок действия операторов (как в [12]). Тогда получаем

$$
\mathscr{P}\left(\stackrel{2}{y}, \stackrel{1}{*}_{y}^{*}\right) K_{x, y}^{\hbar}=\mathscr{P}\left(y, R_{x}^{*}\right) K_{x, y}^{\hbar}
$$

Поскольку множество полиномов всюду плотно в пространстве гладких функций, получаем следующее соотношение:

$$
\alpha_{k}^{\hbar}\left(\stackrel{2}{y}, \stackrel{1}{*}_{y}^{*}\right) K_{x, y}^{\hbar}=\alpha_{k}^{\hbar}\left(y, R_{x}^{*}\right) K_{x, y}^{\hbar}
$$


где $\alpha_{k}^{\hbar}(y, z)$ - “произвольные" действительные гладкие функции, $k=1, \ldots, 2 n$. Будем строить эти функции так, чтобы уравнения (3.1) превратились в уравнения типа Шрёдингера вида (1.3) (роль времени будет играть переменная $y$ ). То есть будем искать функции $\alpha_{k}^{\hbar}(y, z)$ из следующих соотношений:

$$
\alpha_{k}^{\hbar}\left(\stackrel{2}{y}, L_{y}^{*}\right)=i \hbar \frac{\partial}{\partial y_{k}}
$$

Сопрягая эти соотношения относительно евклидовой меры, получим

$$
\alpha_{k}^{\hbar}\left(\stackrel{1}{y}, \stackrel{2}{L}_{y}\right)=-i \hbar \frac{\partial}{\partial y_{k}}
$$

Разложим левую часть этого равенства в асимптотический ряд по степеням параметра $\hbar$. Для этого разложим операторы регулярного представления в соответствии с формулой (2.4) и используя формулы некоммутативного анализа [4], [12]. Получим

$$
\begin{aligned}
\alpha_{k}^{\hbar}\left(\stackrel{1}{y}, \ell_{\hbar}\left(\stackrel{3}{y},-i \hbar \frac{\partial}{\partial y}\right)\right)= & \alpha_{k}^{\hbar}\left(\stackrel{2}{y}, \ell_{\hbar}\left(\stackrel{2}{y},-i \hbar \frac{\partial}{\partial y}\right)\right) \\
& -\left.i \hbar \frac{\partial^{2} \alpha_{k}^{\hbar}}{\partial y_{s} \partial p_{s}}\left(\stackrel{2}{y}, \ell\left(y^{\prime},-i \hbar \frac{\partial}{\partial y}\right)\right)\right|_{y^{\prime}=\stackrel{1}{2}}+\cdots
\end{aligned}
$$

Будем искать функции $\alpha^{\hbar}(y, z)$ в виде асимптотического ряда

$$
\alpha^{\hbar}(y, z)=\alpha^{(0)}(y, z)+\hbar \alpha^{(1)}(y, z)+\cdots
$$

Учитьвая все сказанное, уравнения (3.2) можно переписать в виде асимптотического разложения

$$
\begin{aligned}
& \alpha_{k}^{(0)}(y, \ell(y, p))-\frac{i \hbar}{2} \frac{\partial^{2} \alpha_{k}^{(0)}}{\partial \ell_{j} \partial \ell_{m}}(y, \ell(y, p)) \frac{\partial \ell_{j}}{\partial y_{s}} \frac{\partial \ell_{m}}{\partial p_{s}}-\left.i \hbar \frac{\partial^{2} \alpha_{k}^{(0)}}{\partial y_{s} \partial p_{s}}\left(y, \ell\left(y^{\prime}, p\right)\right)\right|_{y^{\prime}=y} \\
& +i \hbar \mu_{j}(y, p) \frac{\partial \alpha_{k}^{(0)}}{\partial \ell_{j}}(y, \ell(y, p))+\hbar \alpha_{k}^{(1)}(y, \ell(y, p))+O\left(\hbar^{2}\right)=p_{k}
\end{aligned}
$$

Приравнивая в этом соотношении коэффициенты при различных степенях параметра $\hbar$, получаем выражения для коэффициентов асимптотического разложения (3.3)

$$
\begin{aligned}
\alpha_{k}^{(0)}(y, \ell(y, p))= & p_{k} \\
\alpha_{k}^{(1)}(y, \ell(y, p))= & \frac{i}{2} \frac{\partial^{2} \alpha_{k}^{(0)}}{\partial \ell_{j} \partial \ell_{m}}(y, \ell(y, p)) \frac{\partial \ell_{j}}{\partial y_{s}} \frac{\partial \ell_{m}}{\partial p_{s}}-\left.i \frac{\partial^{2} \alpha_{k}^{(0)}}{\partial y_{s} \partial p_{s}}\left(y, \ell\left(y^{\prime}, p\right)\right)\right|_{y^{\prime}=y} \\
& -i \mu_{j}(y, p) \frac{\partial \alpha_{k}^{(0)}}{\partial \ell_{j}}(y, \ell(y, p)) .
\end{aligned}
$$


Отсюда получаем выражения для коэффициентов $\alpha_{1}$

$$
\begin{aligned}
\alpha^{(1)}(y, \ell)= & \frac{i}{2} \frac{\partial^{2} \alpha^{(0)}}{\partial \ell_{j} \partial \ell_{m}}(y, \ell) \frac{\partial \ell_{j}}{\partial y_{s}}\left(y, \alpha^{(0)}(y, \ell)\right) \frac{\partial \ell_{m}}{\partial p_{s}}\left(y, \alpha^{(0)}(y, \ell)\right) \\
& +i \frac{\partial^{2} \alpha^{(0)}}{\partial y_{s} \partial \ell_{j}}(y, \ell) \frac{\partial \ell_{j}}{\partial p_{s}}\left(y, \alpha^{(0)}(y, \ell)\right)-i \mu_{j}\left(y, \alpha^{(0)}(y, \ell)\right) \frac{\partial^{2} \alpha^{(0)}}{\partial \ell_{j}}(y, \ell) .
\end{aligned}
$$

Аналогично, для операторов $R^{*}$ можно выписать разложение

$$
R_{x}^{*}=\ell\left(\stackrel{\omega}{x},-i \hbar \frac{\omega}{\partial x}\right)-\frac{i \hbar}{2} \frac{\partial^{2} \ell}{\partial x \partial p}\left(\stackrel{\omega}{x},-i \hbar \frac{\omega}{\partial x}\right)+i \hbar \mu\left(\stackrel{\omega}{x},-i \hbar \frac{\omega}{\partial x}\right)+\cdots
$$

Поэтому для оператора $\alpha^{\hbar}\left(x, R_{y}^{*}\right)$ имеем

$$
\begin{aligned}
& \alpha^{\hbar}\left(x, R_{y}^{*}\right)=\alpha^{\hbar}\left(y, \ell\left(\stackrel{\omega}{x},-i \hbar \frac{\omega}{\partial x}\right)\right) \\
& \quad+i \hbar\left[-\frac{1}{2} \frac{\partial^{2} \ell_{j}}{\partial x \partial p}\left(\stackrel{\omega}{x},-i \hbar \frac{\partial}{\partial x}\right)-\mu_{j}\left(\stackrel{\omega}{x},-i \hbar \frac{\partial}{\partial x}\right)\right] \frac{\partial \alpha^{\hbar}}{\partial \ell_{j}}\left(y, \ell\left(\stackrel{\omega}{x},-i \hbar \frac{\partial}{\partial x}\right)\right)+\cdots
\end{aligned}
$$

Используя теперь асимптотическое разложение для функции $\alpha^{\hbar}$, получим

$$
\begin{aligned}
& \alpha^{\hbar}\left(x, R_{y}^{*}\right)=\alpha^{(0)}\left(y, \ell\left(\stackrel{\omega}{x},-i \hbar \frac{\omega}{\partial x}\right)\right)+\hbar \alpha^{(1)}\left(y, \ell\left(\stackrel{\omega}{x},-i \hbar \frac{\omega}{\partial x}\right)\right) \\
& \quad+i \hbar\left[-\frac{1}{2} \frac{\partial^{2} \ell_{j}}{\partial x \partial p}\left(\stackrel{\omega}{x},-i \hbar \frac{\partial}{\partial x}\right)-\mu_{j}\left(\stackrel{\omega}{x},-i \hbar \frac{\partial}{\partial x}\right)\right] \frac{\partial \alpha^{(0)}}{\partial \ell_{j}}\left(y, \ell\left(\stackrel{\omega}{x},-i \hbar \frac{\partial}{\partial x}\right)\right)+\cdots
\end{aligned}
$$

Теперь из (3.1) следует

Теорема 3.1. Интегральное ядро $K=K_{x, y}^{\hbar}(u)$ ассоииативного некоммутативного *-произведения функиий на симплектическом пространстве удовлетворяет следующей системе уравнений в частных производных типа Шрёдингера $(k=1, \ldots, 2 n)$ :

$$
i \hbar \frac{\partial}{\partial y_{k}} K_{x, y}^{\hbar}=\left[H_{y k}^{(0)}\left(\stackrel{\omega}{x},-i \hbar \frac{\omega}{\partial x}\right)+\hbar H_{y k}^{(1)}\left(\stackrel{\omega}{x},-i \hbar \frac{\partial}{\partial x}\right)+\cdots\right] K_{x, y}^{\hbar},
$$

әде обозначено

$$
\begin{aligned}
& H_{y}^{(0)}(x, p)=\alpha^{(0)}(y, \ell(x, p)) \\
& H_{y}^{(1)}(x, p)=\alpha^{(1)}(y, \ell(x, p))+i\left[-\frac{1}{2} \frac{\partial^{2} \ell_{j}}{\partial x \partial p}(x, p)-\mu_{j}(x, p)\right] \frac{\partial \alpha^{(0)}}{\partial \ell_{j}}(y, \ell(x, p))
\end{aligned}
$$

а функиии $\alpha^{(0)}$ и $\alpha^{(1)}$ определены в (3.4) и (3.5). 
СлЕДСтвИЕ. Разложсение *-произведения (1.2) $c$ ядром $K_{\hbar}$, определенным в (3.6), по степеням параметра $\hbar$ имеет вид (1.1).

4. ВКБ-приближение для вейлевского произведения. Итак, мы получили систему уравнений (3.6) для интегрального ядра $K^{\hbar}$. Построим приближенное решение полученных уравнений в ВКБ-форме

$$
K^{\hbar}=\exp \left(\frac{i}{\hbar} S\right)(\varphi+O(\hbar))
$$

Для фазы $S$ интегрального ядра и главного слагаемого асимптотического разложения амплитуды $\varphi$ получаются соответственно система уравнений Гамильтона-Якоби

$$
\frac{\partial S}{\partial y_{k}}+H_{y k}^{(0)}\left(x, \frac{\partial S}{\partial x}\right)=0
$$

и система уравнений переноса

$$
\frac{\partial \varphi}{\partial y_{k}}+\left[\frac{1}{2} \operatorname{tr} \frac{\partial}{\partial x}\left(\frac{\partial H_{y k}^{(0)}}{\partial p}\left(x, \frac{\partial S}{\partial x}\right)\right)+\operatorname{tr}\left(\frac{\partial H_{y k}^{(0)}}{\partial p}\left(x, \frac{\partial S}{\partial x}\right)\right) \frac{\partial}{\partial x}+i H_{y k}^{(1)}\left(x, \frac{\partial S}{\partial x}\right)\right] \varphi=0
$$

Начальные условия для этих систем выберем таким образом, чтобы для ядра $K$ были выполнены следующие соотношения:

$$
\begin{aligned}
& \frac{1}{(2 \pi \hbar)^{2 n}} \int K_{x, y}^{\hbar}(u) d x=\delta(u-y) \\
& \frac{1}{(2 \pi \hbar)^{2 n}} \int K_{x, y}^{\hbar}(u) d y=\delta(u-x)
\end{aligned}
$$

(При наличии этих соотношений единичная функция будет двусторонней единицей в *-алгебре). Соотношения (4.4) и (4.5) будут выполнены с точностью $O(\hbar)$ для ядра (4.1), если

$$
\begin{aligned}
& \left.S(u, x, y)\right|_{y=u}=0 \\
& \left.\varphi(u, x, y)\right|_{y=u}=\left|\operatorname{det}\left(\frac{\partial^{2} S}{\partial x \partial y}\right)\right|_{y=u}
\end{aligned}
$$

Систему уравнений (4.2), (4.3) с начальными условиями (4.6), (4.7) будем теперь решать методом характеристик. Характеристиками для этой системы являются решения уравнений

$$
\frac{d P_{j}}{d y_{k}}=-\frac{\partial H_{y k}^{(0)}}{\partial x_{j}}(X, P), \quad \frac{d X_{j}}{d y_{k}}=\frac{\partial H_{y k}^{(0)}}{\partial p_{j}}(X, P)
$$

с начальньми условиями

$$
\left.X_{j}\right|_{y=u}=x_{0 j},\left.\quad P_{j}\right|_{y=u}=0 .
$$

Пусть решение системы Гамильтона (4.8), (4.9) найдено. Тогда решение уравнения Гамильтона-Якоби (4.2) дается обычной формулой. 
Лемма 4.1. Решение уравнения Гамильтона-Якоби $(4.2)(k=1,2)$ с начальным условием (4.6) дается формулой

$$
\begin{aligned}
& S(u, x, y)=\int_{u}^{y} d y_{1}\left\langle P\left(y, x_{0}\right), \frac{\partial X\left(y, x_{0}\right)}{\partial y_{1}}\right\rangle-H_{y 1}^{(0)}\left(X\left(y, x_{0}\right), P\left(y, x_{0}\right)\right) \\
& \quad+\int_{u}^{y} d y_{2}\left\langle P\left(u_{1}, y_{2}, x_{0}\right), \frac{\partial X\left(u_{1}, y_{2}, x_{0}\right)}{\partial y_{2}}\right\rangle-H_{y 2}^{(0)}\left(X\left(u_{1}, y_{2}, x_{0}\right), P\left(u_{1}, y_{2}, x_{0}\right)\right)
\end{aligned}
$$

әде после интегрирования следует подставить $x_{0}=x_{0}(x, y)$ - решение уравнения $x=X\left(x_{0}, y\right)$ относительно $x_{0}$.

Конечно, здесь и в следующей лемме 4.2 предполагается гладкая разрешимость уравнения $x=X\left(x_{0}, y\right)$ относительно $x_{0}$. Функция $S(4.10)$ корректно определена только в соответствуюшей подобласти пространства $\mathfrak{X} \times \mathfrak{X} \times \mathfrak{X}$ (см. подробнее [8]).

Оказьвается, что эта конструкция фазы $S$ интегрального ядра $K^{\hbar}$ имеет простой геометрический смысл: она выражается через площадь треугольника в пространстве $\mathfrak{X}$. В [8] показано, что фаза интегрального ядра ассоциативного некоммутативного *-произведения символов, определенная формулой (4.10), совпадает с площадью мембраны, образованной тремя “эфирными” геодезическими со средними точками $u, x, y$, что реализует общую гипотезу [13], [14].

Система уравнений переноса (4.3) также легко решается.

ЛЕмма 4.2. Решение системы уравнений переноса $(4.3)(k=1,2)$ с начальным условием (4.7) дается формулой

$$
\begin{aligned}
\varphi(u, x, y)=\frac{\left|\operatorname{det}\left(\partial^{2} S / \partial x \partial y\right)\right|_{y=u}}{\sqrt{\left|\operatorname{det}\left(\partial X / \partial x_{0}\right)\right|}} \exp \left(-i \int_{u}^{y} d y_{1} H_{y 1}^{(1)}\left(X\left(y, x_{0}\right), P\left(y, x_{0}\right)\right)\right) \\
\quad \times \exp \left(-i \int_{u}^{y} d y_{2} H_{y 2}^{(1)}\left(X\left(u_{1}, y_{2}, x_{0}\right), P\left(u_{1}, y_{2}, x_{0}\right)\right)\right)
\end{aligned}
$$

где после интегрирования следует подставить $x_{0}=x_{0}(x, y)$ как в (4.10).

Эта формула для главного члена асимптотики амплитуды ядра $K^{\hbar}$ отличается от найденной в [8] множителями, содержащими первую квантовую поправку $H^{(1)}$, заданную в (3.7), (3.5). При этом гамильтониан $\mathscr{H}^{\hbar}$ в основном динамическом уравнении (1.3) уже не предполагается, в отличие от [8], вещественным в младших членах по $\hbar$.

Можно вьписать также уравнения для следующих поправок к главному слагаемому асимптотического разложения амплитуды $\varphi$, и таким образом построить решение системы (3.6) с любой степенью точности по $ћ$ вне зон фокусировки.

Появление фокальных точек, где асимптотика ядра $K^{\hbar}$ уже не имеет ВКБ-вид (4.1), а должна задаваться какой-то модификацией канонического оператора Маслова [15], определяется обращением в нуль якобиана $\operatorname{det}\left(\partial X / \partial x_{0}\right)$. Появление фокальных точек над плоскостью Лобачевского было продемонстрировано в [16]. 
5. Пример. Для иллюстрации полученных результатов рассмотрим следующий несложньй пример. В качестве исходного многообразия возьмем правую полуплоскость $x_{1}>0$ двумерной плоскости $\left(x_{1}, x_{2}\right)$, снабженную пуассоновой структурой

$$
\Psi^{12}(x)=-2 x_{1}^{3 / 2}
$$

Считаем, что координаты $x_{1}, x_{2}$ аффинные. Соответствующая связность $(2.2)$ здесь имеет только два отличные от нуля символа Кристофеля:

$$
\Gamma_{11}^{1}=-\frac{1}{x_{1}}, \quad \Gamma_{12}^{2}=-\frac{1}{2 x_{1}} .
$$

Кривизна данной связности является ковариантно постоянной, т.е. $\nabla R=0$ и, таким образом, наше пространство симметрическое.

Система уравнений Шрёдингера (3.6) с учетом формул $(2.5),(2.6),(3.5)$ и (3.7) будет иметь вид

$$
\begin{aligned}
i \hbar \frac{\partial}{\partial y_{1}} K_{x, y}^{\hbar} & =\left[\frac{\ell_{2}-y_{2}}{y_{1} \sqrt{\ell_{1}}}-\frac{i \hbar}{2}\left(\frac{3}{y_{1}}+\frac{2 \ell_{1}+x_{1}}{y_{1}\left(\ell_{1}+x_{1}\right)}\right)\right] K_{x, y}^{\hbar}, \\
i \hbar \frac{\partial}{\partial y_{2}} K_{x, y}^{\hbar} & =\frac{y_{1}-\ell_{1}}{y_{1} \sqrt{\ell_{1}}} K_{x, y}^{\hbar}
\end{aligned}
$$

где $\ell=\ell\left(x,-i \hbar \frac{\partial}{\partial x}\right)$, а левые отображения сокращения заданы формулами

$$
\begin{aligned}
& \ell_{1}(x, p)=x_{1}+\frac{1}{2} x_{1} p_{2}\left(x_{1} p_{2}-\sqrt{x_{1}\left(x_{1} p_{2}^{2}+4\right)}\right) \\
& \ell_{2}(x, p)=x_{2}-\frac{1}{2} x_{1} p_{1}\left(x_{1} p_{2}-\sqrt{x_{1}\left(x_{1} p_{2}^{2}+4\right)}\right) .
\end{aligned}
$$

Применяя теперь к этому уравнению описанный вьше метод ВКБ, т.е. решая соответствующие уравнения Гамильтона-Якоби (4.2) и переноса $(4.3)$, получим для ядра $K_{x, y}^{\hbar}(u)$ следующее выражение:

$$
K_{x, y}^{\hbar}(u)=\frac{x_{1}+y_{1}}{2 x_{1}^{2} y_{1}^{2}} \exp \left(\frac{i}{\hbar} \frac{u_{2}\left(y_{1}-x_{1}\right)+x_{2}\left(u_{1}-y_{1}\right)+y_{2}\left(x_{1}-u_{1}\right)}{\sqrt{x_{1} y_{1} u_{1}}}\right)+O(\hbar)
$$

Покажем теперь, что в данном примере остаток $O(\hbar)$ на самом деле равен нулю.

Построим семейство самосопряженных операторов $\mathbf{S}_{x}$, которое задает гомоморфизм $f \rightarrow \mathrm{Op}(f)$ алгебры символов в алгебру операторов по следующей формуле:

$$
\mathrm{Op}(f)=\int f(x) \mathbf{S}_{x} d x
$$

Мы строим $\mathbf{S}_{x}$ исходя из того, что в аффинных координатах должно быть Ор $(f)=$ $f\left(\hat{x}_{1}, \hat{x}_{2}\right)$, где в правой части $\hat{x}_{1}, \hat{x}_{2}$ симметризованы по Вейлю и удовлетворяют соотношениям

$$
\left[\hat{x}_{1}, \hat{x}_{2}\right]=-i \hbar \Psi^{12}\left(\hat{x}_{1}, \hat{x}_{2}\right)
$$


Тогда семейство $\mathbf{S}_{x}$ может быть представлено в виде

$$
\mathbf{S}_{x}=\frac{1}{(2 \pi \hbar)^{2}} \int \exp \left(-\frac{i}{\hbar}\left(\tau_{1} x_{1}+\tau_{2} x_{2}\right)\right) \exp \left(\frac{i}{\hbar}\left(\tau_{1} \hat{x}_{1}+\tau_{2} \hat{x}_{2}\right)\right) d \tau_{1} d \tau_{2}
$$

В гильбертовом пространстве $L^{2}(\mathbb{R})$ выберем следующую реализацию коммутационных соотношений:

$$
\hat{x}_{1}=\exp t, \quad \hat{x}_{2}=-i \hbar \exp \frac{t}{2}\left(2 \frac{\partial}{\partial t}+\frac{1}{2}\right) \text {. }
$$

Подставляя эти операторы в формулу (5.3), легко вычислим семейство $\mathbf{S}_{x}$. Оно действует по формуле

$$
\left(\mathbf{S}_{x} \psi\right)(t)=\frac{1}{x_{1}^{3 / 2}} \exp \left(\frac{i}{\hbar} \frac{\exp (t / 2)-x_{1} \exp (-t / 2)}{x_{1}} x_{2}\right) \psi\left(-t+2 \log x_{1}\right), \quad \psi \in L^{2}(\mathbb{R})
$$

Введем также “двойственное” семейство операторов $\widetilde{\mathbf{S}}_{x}$ так, чтобы было вьполнено соотношение

$$
\operatorname{tr}\left(\widetilde{\mathbf{S}}_{x} \mathbf{S}_{y}\right)=\delta(x-y)
$$

Нетрудно убедиться, что

$$
\begin{aligned}
\left(\widetilde{\mathbf{S}}_{x} \psi\right)(t)= & \frac{1}{2 x_{1}^{1 / 2}}\left(\exp \frac{t}{2}+x_{1} \exp \left(\frac{-t}{2}\right)\right) \\
& \times \exp \left(\frac{i}{\hbar} \frac{\exp (t / 2)-x_{1} \exp (-t / 2)}{x_{1}} x_{2}\right) \psi\left(-t+2 \log x_{1}\right) .
\end{aligned}
$$

Символ оператора (5.2) восстанавливается по формуле

$$
f(x)=\operatorname{tr}\left(\widetilde{\mathbf{S}}_{x} \mathrm{Op}(f)\right) .
$$

Отсюда получаем, что ядро квантового произведения имеет вид следа композиции

$$
K_{x, y}^{\hbar}(u)=\operatorname{tr}\left(\widetilde{\mathbf{S}}_{u} \mathbf{S}_{x} \mathbf{S}_{y}\right)=\frac{x_{1}+y_{1}}{2 x_{1}^{2} y_{1}^{2}} \exp \left(\frac{i}{\hbar} \frac{u_{2}\left(y_{1}-x_{1}\right)+x_{2}\left(u_{1}-y_{1}\right)+y_{2}\left(x_{1}-u_{1}\right)}{\sqrt{x_{1} y_{1} u_{1}}}\right)
$$

т.е. остаток в (5.1) на самом деле отсутствует.

\section{СПИСОК ЦИТИРОВАННОЙ ЛИТЕРАТУРЫ}

[1] Bayen F., Flato M., Fronsdal C., Lichnerowicz A., Sternheimer D. Deformation theory and quantization // Ann. Physics. 1978. V. 111. P. 61-151.

[2] Omori H., Maeda Y., Yoshioka A., Weyl manifolds and deformation quantization // J. Adv. Math. 1997. V. 85. P. 224-255. 
[3] Карасев М. В., Маслов В. П. Асимптотическое и геометрическое квантование // УМН. 1984. Т. 39. №6. С. 115-173.

[4] Карасев М. В., Маслов В. П. Нелинейные скобки Пуассона. Геометрия и квантование. М.: Наука, 1991.

[5] Карасев М.В. Аналоги объектов теории групп Ли для нелинейных скобок Пуассона // Изв. АН СССР. Сер. матем. 1986. Т. 50. № 3. С. 508-538.

[6] Weinstein A. Symplectic groupoids and Poisson manifolds // Bull. Amer. Math. Soc. 1987. V. 16. P. 101-104.

[7] Карасев М.В. Квантование нелинейных скобок Ли-Пуассона в квазиклассическом приближении // Препринт № ITP-85-72P. Киев: ИТФ, 1985.

[8] Karasev M. Quantization and intrinsic dynamics // Asymptotic Methods For Wave And Quantum Equations / ed. M. Karasev et al. Advances in Math. Sci. Providence, RI: Amer. Math. Soc., 2003.

[9] Григорьев О.Н., Карасев М.В.Внутренняя квантовая динамика и ее операторное представление над плоскостью с нестандартной связностью // Russ. J. Math. Phys. 2003. V. 10. № 4. P. 422-435.

[10] Karasev M. V. Advances in quantization: quantum tensors, explicit star-products, and restriction to irreducible leaves // Diff. Geometry Appl. 1998. V. 9. P. 89-134.

[11] Karasev M. V. Maslov quantization conditions in higher cohomology and analogs of notions developed in Lie theory for canonical fiber bundles of symplectic manifolds // Deposited at VINITI (March 12, 1982, № 1092-82, 1093-82). М.: МИЭМ, 1981.

[12] Маслов В. Операторные методы. М.: Наука, 1973.

[13] Березин Ф. А. Квантование // Изв. АН СССР. Сер. матем. 1974. Т. 38. № 5. С. 1109-1165.

[14] Weinstein A. Traces and triangles in symmetric symplectic spaces // Contemp. Math. 1994. V. 179. P. 262-270.

[15] Маслов В. П. Теория возмущений и асимптотические методы. М.: Изд-во Моск. ун-та, 1965.

[16] Tuynman G., Rios P. Weyl quantization from geometric quantization // Preprint. Lille, 1999.

Московский государственный институт электроники и математики

Поступило

E-mail : karasev@miem.edu.ru

17.10 .2003

Исправленный вариант

11.03.2004 